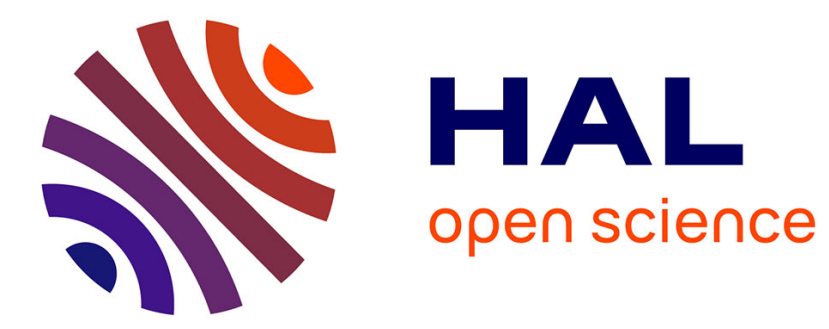

\title{
Cross Recurrence Plot Analysis Based Method For TDOA Estimation Of Underwater Acoustic Signals
}

Olivier Le Bot, Jerome I. Mars, Cedric Gervaise, Yvan Simard

\section{To cite this version:}

Olivier Le Bot, Jerome I. Mars, Cedric Gervaise, Yvan Simard. Cross Recurrence Plot Analysis Based Method For TDOA Estimation Of Underwater Acoustic Signals. CAMSAP 2015 - 6th IEEE workshop on Computational Advances in Multi-Sensor Adaptive Processing, IEEE Dec 2015, Cancun, Mexico. pp.325-328. hal-01245245

\section{HAL Id: hal-01245245 \\ https://hal.science/hal-01245245}

Submitted on 18 Dec 2015

HAL is a multi-disciplinary open access archive for the deposit and dissemination of scientific research documents, whether they are published or not. The documents may come from teaching and research institutions in France or abroad, or from public or private research centers.
L'archive ouverte pluridisciplinaire HAL, est destinée au dépôt et à la diffusion de documents scientifiques de niveau recherche, publiés ou non, émanant des établissements d'enseignement et de recherche français ou étrangers, des laboratoires publics ou privés. 


\section{Cross Recurrence Plot Analysis Based Method For TDOA Estimation Of Underwater Acoustic Signals}

\author{
Olivier Le Bot, Jérôme I. Mars \\ Univ. Grenoble Alpes, GIPSA-Lab \\ Univ. Grenoble Alpes, CNRS \\ F-38000 Grenoble, France \\ Olivier.le-bot@gipsa-lab.grenoble-inp.fr \\ Jerome.mars@gipsa-lab.grenoble-inp.fr
}

\author{
Cédric Gervaise \\ Foundation of Grenoble-INP \\ 46 Avenue Félix Viallet \\ 38031 Grenoble, France \\ cedric.gervaise@chorusacoustics.com
}

\author{
Yvan Simard \\ Marine Science Institute \\ University of Quebec at Rimouski \\ 300 Allée des Ursulines \\ Rimouski, QC G5L 3A1, Canada \\ Yvan.Simard@dfo-mpo.gc.ca
}

\begin{abstract}
In this paper, we propose to use cross recurrence plot analysis (CRPA) to estimate the time-difference of arrival (TDOA) of underwater acoustic signals arriving on an array of hydrophones. Instead of considering the signal as a whole to estimate the TDOA, like classical methods do, we first detected the series of samples that look alike on each pair of hydrophones of the array by using cross-recurrence plot analysis. The TDOA is then estimated by relying only on these common sample series. The TDOA estimator is based on quantification measures specifically designed for CRPA. The proposed method is successfully validated on real data containing frequency-modulated sounds from beluga whales.
\end{abstract}

\section{INTRODUCTION}

Various applications, including military surveillance, bioacoustics or environmental monitoring to name but a few, make use of array of hydrophones to estimate the direction of arrival of underwater acoustic sources. Such an estimation can be made through two broad-classes of methods:

1) Estimation of the time-difference of arrival (TDOA) of a signal received at spatially separated sensors followed by a spatial-inversion scheme.

2) Space-time processing like beamforming or spatial spectral estimation (Capon, MUSIC, etc.)

In the first class cited above, the estimation of the TDOA generally relies on the cross-correlation, which is a classical and easy-to-use signal processing function even for non-specialists in signal processing. Therefore, the crosscorrelation is extensively used in bio-acoustics for the localization of cetaceans for instance [1]-[3]. Thorough state-of-the-art reviews of other TDOA estimators can be found in [3]-[5].

A common point to all TDOA estimators found in the literature is that they consider the signal as a whole. However, underwater acoustic sources are immersed in a noisy environment and the propagation through the oceanic canal modifies the signal by introducing amplitude and phase modulations, spectral distortions and reverberation. Thus, the signal received can be very different from one hydrophone to the other and only few series of samples remain similar on all the sensors.

Based on these observations, we propose a new approach to estimate the TDOA. It is derived from the field of non-linear analysis of dynamical systems and is called Cross-Recurrence Plot analysis (CRPA) [6]. First, CRPA aims to identify series of samples that are similar on two hydrophones. The result of the CRPA can be seen as a binarized or thresholded similarity matrix. The TDOA can then be estimated from this latter using some specific measures especially dedicated to the quantification the information contained in the CRPA matrices. CRPA has already been used in the past for synchronization of dynamical systems and time-scale alignment of data series [6], [7]. To our knowledge it is the first time that CRPA is used with acoustic signals for TDOA estimation.

Section II gives the basics of Recurrence Plot Analysis (RPA) and Cross-Recurrence Plot Analysis (CRPA). Then some quantification measures are proposed to estimate the TDOA from the CRPA. Section III tests the proposed quantification measures on simulated signals and briefly discusses their reliability for TDOA estimation. The proposed method is finally validated on real signals, namely frequency-modulated signals produced by beluga whales. This validation shows that our method succeed when the cross-correlation does not.

\section{METHOD}

\section{A. Recurrence Plot Analysis (RPA)}

Recurrence plot analysis (RPA) was introduced to study the dynamical behavior of complex systems and are aimed at visualizing the recurrence of their phase space trajectory [9]. Transforming a data sequence to a recurrence plot representation involves three main steps.

First, the trajectory of the measured signal $x$ in the phase space is reconstructed using the time-delay embedding method [10], [11]. This trajectory is described by a series of vectors given by:

$$
\overrightarrow{x_{m}(i)}=[x(i), x(i+\tau), \ldots, x(i+(m-1) \tau)]
$$

with $m \in \mathbb{N}^{*}$ the embedding dimension of the phase space, $\tau \in$ $\mathbb{N}^{*}$ a delay (not related to the TDOA), $i=\left\{1 \ldots N_{x}-(m-1) \tau\right\}$ the sample index and $N_{x}$ the number of samples of $x$.

The second step consists of measuring the degree of similarity between two vectors of the phase space trajectory: $\overrightarrow{x_{m}(i)}$ and $\overrightarrow{x_{m}(j)}$. Calculating the similarity between all of the possible pairs of phase space vectors gives the similarity matrix defined by:

$$
d(i, j)=\operatorname{Sim}\left(\overrightarrow{x_{m}(i)}, \overrightarrow{x_{m}(j)}\right)
$$


where $\operatorname{Sim}(.,$.$) is the function that is chosen to study the$ likeness of the phase space vectors. A lot of different mathematical functions can be used for this step [8]. The Euclidean norm is mostly used for this purpose by the recurrence plot community [7]. In this paper, we will use the dot product which is a particular case of cross-correlation, classically used for TDOA estimation. We could say that our similarity matrix is obtained through a kind of piecewise cross-correlation.

Finally, the recurrence plot is obtained by comparing each coefficient of the similarity matrix to a threshold $\varepsilon$. Therefore, the recurrence plot is a binary matrix, where the coefficient of index $(i, j)$ is 1 if $\overrightarrow{x_{m}(i)}$ and $\overrightarrow{x_{m}(j)}$ are considered as similar, and is 0 otherwise. In a mathematical formalism, the recurrence plot is defined as follow:

$$
R P A(i, j)=\Theta\left(\operatorname{Sim}\left(\overrightarrow{x_{m}(i)}, \overrightarrow{x_{m}(j)}\right)-\varepsilon\right)
$$

where $\Theta$ is the Heaviside function. The RPA matrix can be represented by a binary image. A summary of the guidelines to follow for choosing appropriate values for $\tau, m$ and $\varepsilon$ parameters can be found the the RPA literature [7].

\section{B. Cross Recurrence Plot Analysis (CRPA)}

RPA can be extended to the bi-variate case to study the dependencies between two different systems by comparing their states [6]. This approach is called Cross-Recurrence Plot Analysis (CRPA) and follows the same three steps of the previously described RPA [6].

Considering two measured signals $x$ and $y$ recorded on two different sensors, the phase space trajectories of each signal are simultaneously reconstructed with the same embedding dimension $m$ and delay $\tau$. Their respective phase space vectors are given by:

$$
\begin{aligned}
& \overrightarrow{x_{m}(i)}=[x(i), x(i+\tau), \ldots, x(i+(m-1) \tau)] \\
& \overrightarrow{y_{m}(j)}=[y(j), y(j+\tau), \ldots, y(j+(m-1) \tau)]
\end{aligned}
$$

and the similarity matrix is now defined by:

$$
d(i, j)=\operatorname{Sim}\left(\overrightarrow{x_{m}(i)}, \overrightarrow{y_{m}(j)}\right)
$$

As for the RPA, the CRPA is obtained through the comparison of each coefficient of the similarity matrix to a threshold. It is then defined as follow:

$$
C R P A(i, j)=\Theta\left(\operatorname{Sim}\left(\overrightarrow{x_{m}(i)}, \overrightarrow{y_{m}(j)}\right)-\varepsilon\right)
$$

\section{Recurrence Quantification Analysis (RQA)}

Depending on the dynamical behavior of the signal (i.e. random, periodic, etc.), the binary matrices obtained form the RPA and CRPA exhibit some patterns as shown on Fig. 1.

For example, single isolated recurrence points appear when states are rarely repeated and do not last in time. Such a case happen on Fig. 1 when comparing two state space vectors associated with noise samples for instance. On the contrary, when different parts of the measured signals have a similar local evolution, then a set of several consecutive state space vectors are considered as similar to another set and the CRPA (resp. RPA) gives a diagonal line, i.e. $C R P A(i+k, j+k)=1$ (for $k=1 \ldots l$ ). Also, when a given waveform is repeated with a certain periodicity, then a set of several diagonal lines parallel to each others appears on the image, as it is the case on Fig. 1 because of the repetitions of the oscillations due to the cosine function within the received signal. At last, Fig. 1 shows that the position of the pattern associated to the cosine changes depending on the TDOA between both receivers. Different measures named as recurrence quantification analysis (RQA) [6], [7] can be used to quantify patterns on the CRPA images and in particular to estimate the TDOA. In this paper we investigate three of these measures.

All the following RQA are based on recurrences forming diagonal lines parallel to the main diagonal. They are calculated for each diagonal of the CRPA matrix taken separately, and are therefore functions of the index of the diagonal $t \in[-T, \ldots, T]$, where $t=0$ corresponds to the main diagonal, $t>0$ are the diagonals above the main diagonal and $t<0$ are the diagonals below the main diagonal. $T$ is the maximum allowable TDOA value (expressed in sample) with respect to the geometry of the array.

The first RQA is called the $t$-recurrence rate and is a classical RQA in the RPA community [6]. It is defined as:

$$
R R(t)= \begin{cases}\frac{1}{N+t} \sum_{i=1}^{N+t} \operatorname{CRPA}(i-t, i) & (t<0) \\ \frac{1}{N-t} \sum_{i=1}^{N-t} \operatorname{CRPA}(i, i+t) & (t \geq 0)\end{cases}
$$

where $N$ is the total number of lines (resp. columns) of the CRPA matrix. This RQA gives the probability of occurrence of similar waveform in both signals with a given time-delay $t$. The greater the number of recurrences in the diagonal $t$ is, the greater the value of $R R(t)$ is.

$R R(t)$ only relies on the binary matrix obtained with the CRPA and do not take into account all the information contained in the similarity matrix $d(i, j)$ given by Eq. (6) that might be valuable. Therefore, we introduce two additional RQA measures that also consider values obtained in the similarity matrix $d(i, j)$.

The second RQA measure is the sum of all similarity values $d(i, j)$ from diagonal $t$ that have lead to recurrence points (i.e $C R P A(i, j)=1)$ in the CRPA matrix. It is defined as:

$$
S S(t)= \begin{cases}\sum_{i=1}^{N+t} C R P A(i-t, i) \odot d(i-t, i) & (t<0) \\ \sum_{i=1}^{N-t} C R P A(i, i+t) \odot d(i, i+t) & (t \geq 0)\end{cases}
$$

where $\odot$ is the Hadamard product.

The last RQA measure is a normalized version of the previous metric and is given by:

$N S(t)= \begin{cases}\frac{1}{N+t} \sum_{i=1}^{N+t} \operatorname{CRPA}(i-t, i) \odot d(i-t, i) & (t<0) \\ \frac{1}{N-t} \sum_{i=1}^{N-t} \operatorname{CRPA}(i, i+t) \odot d(i, i+t) & (t \geq 0)\end{cases}$ 


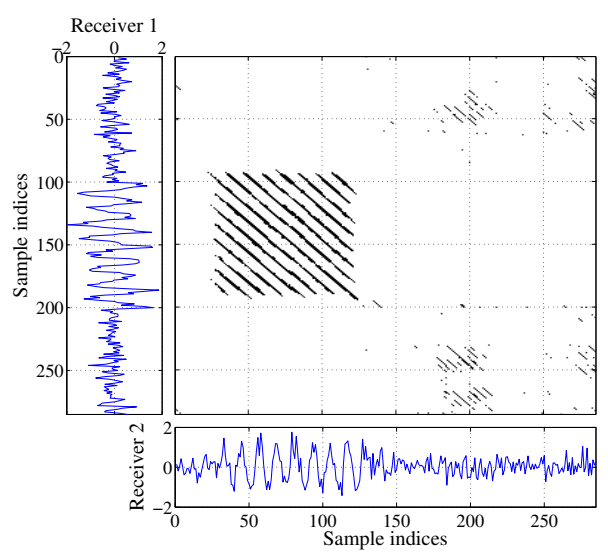

(a) TDOA $=-70$ samples

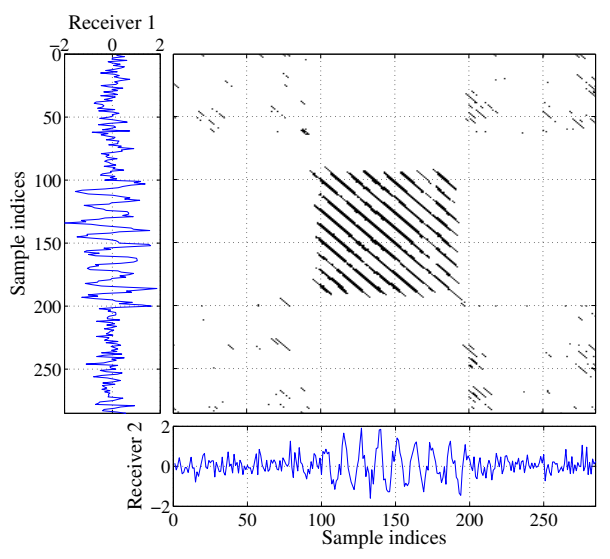

(b) TDOA $=0$ samples

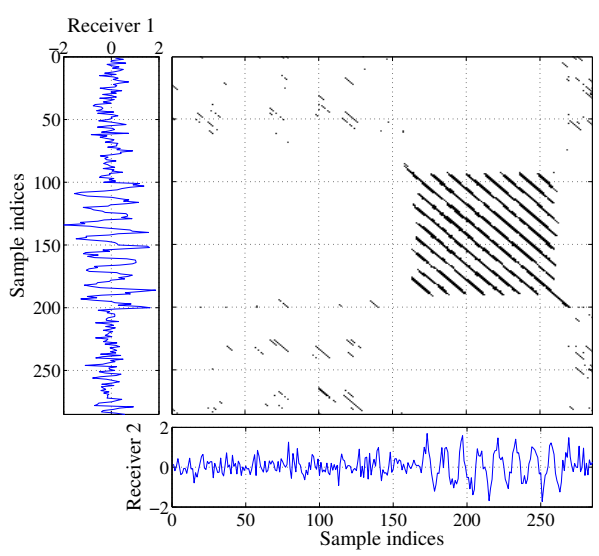

(c) $\mathrm{TDOA}=+70$ samples

Fig. 1: Examples of CRPA matrices in the case of a 100-samples cosine mixed with a 300-samples additive white Gaussian noise received by two sensors with 3 different TDOA values: a) TDOA $=-70$ samples; b) TDOA $=0$ samples; c) TDOA $=+$ 70 samples. Signal to noise ratio is $6 \mathrm{~dB}$. CRPA is built with $\mathrm{m}=16, \tau=1, \operatorname{Sim}(.,)=$. dot product, $\varepsilon=0.075$.

As for $R R(t), S S(t)$ and $N S(t)$ are high if the number of recurrence in diagonal $t$ and if the associated coefficients of the similarity matrix are both high.

\section{Time-difference of arrival estimation}

For the three investigated RQA measures, we said that their values would be high if similar waveform are found for both signals with a given time-delay $t$. Therefore, the estimated value for the TDOA is given by:

$$
\widehat{T D O A}=t \text { such that } \underset{t}{\operatorname{argmax}}(R Q A(t))
$$

where $R Q A(t)$ is either $R R(t), S S(t)$ or $N S(t)$.

\section{RESUlTS}

The proposed TDOA estimator is validated on real underwater acoustic data that were recorded in the Gulf of Saint-Lawrence (Canada) with an array of four hydrophones linked to a data acquisition board connected with a PC. Data were digitized on .wav files. Hydrophones were spaced from 145 to $240 \mathrm{~m}$. A sound speed of $1444 \mathrm{~m} \cdot \mathrm{s}^{-1}$ was used to calculate the maximum allowable TDOA. Prior to the computation of CRPA, data were filtered between 1200 $\mathrm{Hz}$ and $5000 \mathrm{~Hz}$ and down-sampled at $12 \mathrm{kHz}$. Within this frequency band, the database contains a high number of sounds produced by beluga whales, especially frequency-modulated signals. Fig. 2 (left column) shows an example of beluga whale vocalization received by two hydrophones of the array, with obvious differences between both signals in term of amplitude, waveform distortion and background noise level.

The TDOA was estimated with the proposed method. The RQA measure $S S(t)$ appeared to be the most reliable estimator as it gives significantly a higher number of correct TDOA estimates than $N S(t)$ and $R R(t)$. Only the results of this particular RQA are presented in the article. In order to assess the validity of the estimated $\widehat{T D O A}$, we have re-synchronized both received signals by compensating the TDOA by its estimate $\widehat{T D O A}$ (Fig. 2-right column). When looking at the spectrograms after compensation of TDOA, the estimate found by our method seems acceptable. With a close look on the waveform we could notice that both signals are now beginning at the same time. Moreover their oscillations are now perfectly synchronized, which validates the value found for $\widehat{T D O A}$. To show the benefit of the proposed method, we have also estimated the TDOA with the cross-correlation function. As shown on Fig. 2 (central column), when we compensate for the delay between both signals with the estimated TDOA found by the cross-correlation, we clearly see that this latter fails to give a good estimate.

\section{CONCLUSION}

In this article we have proposed a new method for estimating the TDOA of acoustic signals arriving on an array. Instead of considering the signal as a whole, like the classical methods do, we have relied on an analysis method called CrossRecurrence Plot Analysis, which splits the received signals in small pieces and then performs a similarity analysis between these pieces to detect the segments of the waveform that look alike on two hydrophones of the array. When these segments are identified, the TDOA is then estimated by using only these parts of the waveform that are common on both hydrophones. For this latter task we have proposed three quantification measures. The proposed method was successfully validated on real underwater acoustic signals. Tests on additional signals not presented here have shown that it could give reliable TDOA estimate in cases where the cross-correlation function fails.

\section{ACKNOWLEDGMENT}

This work was supported by DGA through the postdoctoral scholarship of O. Le Bot and project ANR-12-ASTR-0021-03.

\section{REFERENCES}

[1] P. Giraudet, H. Glotin, "Real-time 3D tracking of whales by echorobust precise TDOA estimates with a widely-spaced hydrophone array", Applied Acoustics, vol. 67, pp. 1106-1117, 2006 

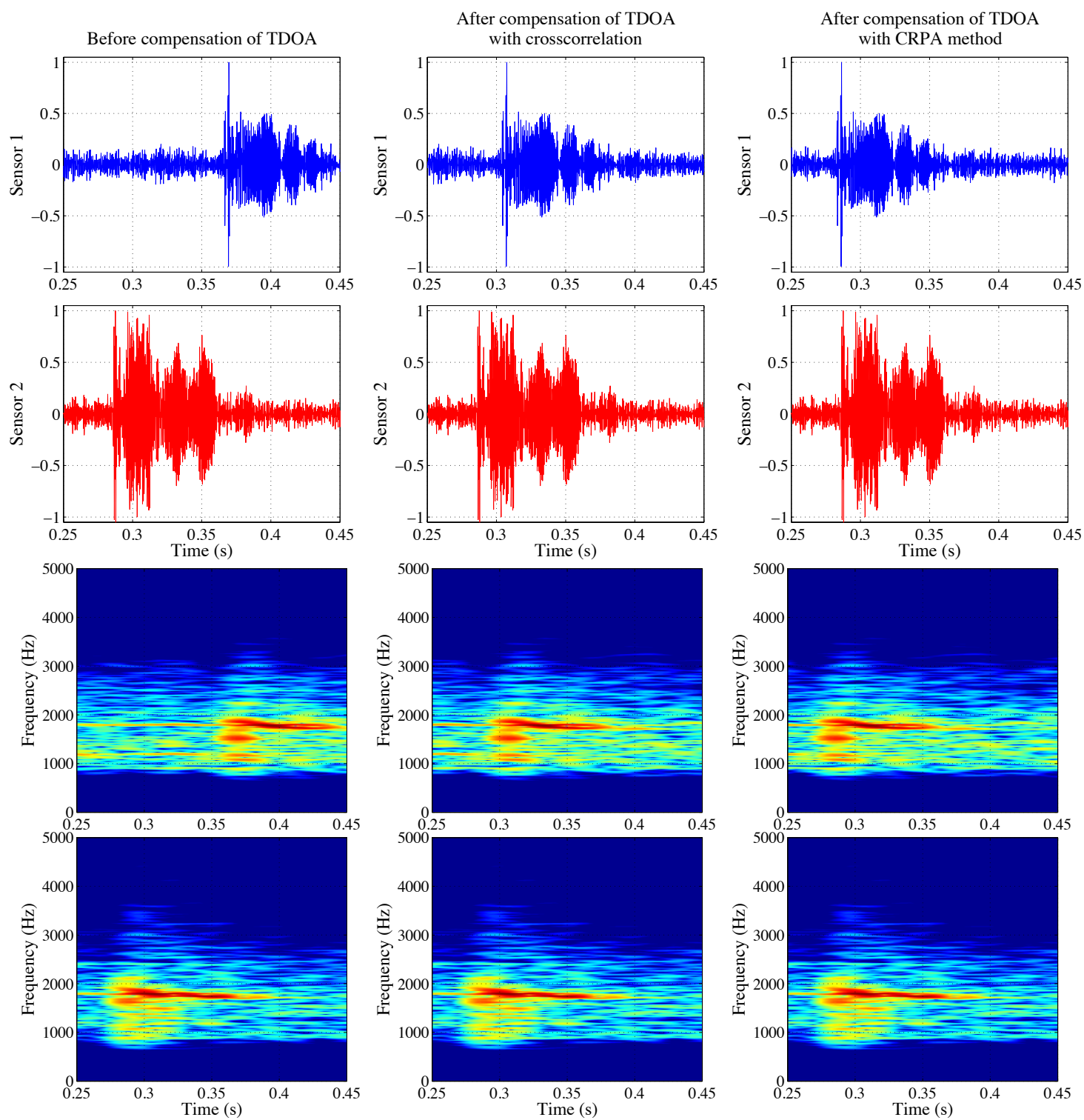

Fig. 2: Example of beluga whale vocalizations received by two hydrophones of the array and their respective spectrogram. Left column shows both original signals as they arrived on the array. Central column shows signals after both signals have been time-aligned with the $\widehat{T D O A}$ value estimated by the classical cross-correlation function. Right column shows signals after they have been time-aligned with the $\widehat{T D O A}$ value estimated with the $S S$ RQA. CRPA was built with $\mathrm{m}=16, \tau=1, \varepsilon=0.075$.

[2] Y. Simard, N. Roy, ’Detection and localization of blue and fin whales from large-aperture autonomous hydrophone arrays: a case study from the St. Lawrence estuary", Canadian Acoustics, vol. 36 (1), pp. 104-110, 2008.

[3] L. Houégnigan, S. Zaugg, M. van der Schaar, M. André, "Spacetime and hybrid algorithms for the passive acoustic localisation of sperm whales and vessels", Applied Acoustics, vol. 71, pp. 1000-1010, 2010.

[4] C. Knapp, G.G. Carter, 'The generalized correlation method for estimation of time delay", IEEE Transactions on Acoustics, Speech and Signal Processing, vol. 24 (4), pp. 320-327, 1976.

[5] J. Chen, J. Benesty, Y. Huang, "Time delay estimation in room acoustic environments: an overview", EURASIP Journal on applied signal processing, pp. 1-19, 2006.

[6] N. Marwan, "Encounters with neighbours: current developments of concepts based on recurrence plots and their applications.", PhD Thesis, University of Potsdam (Germany), 2003.
[7] N. Marwan, M.C. Romano, M. Thiel, J. Kurths, "Recurrence plots for the analysis of complex systems", Physics Reports, vol. 438 (5), pp. 237-329, 2007.

[8] O. Le Bot, C. Gervaise, J.I. Mars, "Similarity matrix analysis and divergence measures for statistical detection of unknown deterministic signals hidden in additive noise.", Physics Letters A, In Press, 2015.

[9] J. P. Eckmann, S.O. Kamphorst, D. Ruelle, "A new graphical tool for measuring the time constancy of dynamical systems is presented and illustrated with typical examples", Europhysics Letters, vol. 4 (91), pp. 973-977, 1987.

[10] N. H. Packard, J.P. Crutchfield, J.D. Farmer, R.S. Shaw, "Geometry from a time series", Physical Review Letters, vol. 45 (9), pp. 712-716, 1980.

[11] F. Taken, "Detecting strange attractors in turbulence", Dynamical systems and turbulence, Lecture Notes in Mathematics, vol. 898, pp. 366381,1981 